\title{
Bismuth Toxicity Masquerading as Alzheimer's Dementia
}

William K. Summers

Alzheimer's Clinic of Albuquerque, 201

Cedar, SE, Suite 404, Albuquerque, NM

87106-4924, USA

Tel: + 1505 843-8331; Fax: +1 505 281-

7665

\begin{abstract}
A 76-year old white married female had rapid onset dementia with myoclonus and was admitted to an HMO hospital where she was initially diagnosed as Alzheimer's disease. The physicianhusband suggested that the condition might be due to the Pepto-Bismol which she had taken chronically to control her irritable bowel syndrome. An elevated serum bismuth level of $242 \mu \mathrm{g} / \mathrm{L}$ (normal is $5 \mu \mathrm{g} / \mathrm{L}$ ) established bismuth toxicity as the cause of the dementia. With treatment the patient returned to a normal mental state. The possibility of bismuth encephalopathy needs to be considered in the differential diagnosis of possible Alzheimer's dementia.
\end{abstract}

\section{INTRODUCTION}

Bismuth was used in medicinal salves as early as 1733 and internally for treatment of spasmodic bowels by the 1790s. Bismuth preparations are generally considered very safe and are often taken without knowledge of possible, albeit rare, neurotoxicity. Bierer's review of bismuth subsalicylate [Pepto-Bismol] states that there have been no cases of neurotoxicity in the United States (1). However, Gordon et al. reported a case of a 54-year old man with suspected bismuth encephalopathy who had taken between 4-16 oz of Pepto-Bismol per day (1.04-4.16 mg/day of bismuth) intermittently over many years to control gastrointestinal upset and diarrhea (2). Unfortunately, the diagnosis was not suspected until the 35th day after the onset of his confusion, and the serum bismuth level was only $3.6 \mu \mathrm{g} / \mathrm{L}$ in the serum. Typically bismuth encephalopathy does not occur without serum levels above 150 $\mu \mathrm{g} / \mathrm{L}$ (7). We report here the case of bismuth encephalopathy with a serum bismuth level of $242 \mu \mathrm{g} / \mathrm{L}$ which was initially incorrectly diagnosed as Alzheimer's disease.

\section{CASE REPORT}

On February 29, 1996, a 76-year old Caucasian female was admitted to a local HMO hospital with a one-week history of progressive confusion, poor appetite, disturbed sleep, and muscle twitching. Her physician-husband noted that she became lost in their retirement complex when attempting to do the laundry and that she complained of feeling like "coming out of a binge". She would take up to an hour to figure out how to put on her pajamas. Pertinent negatives included no history of fever, stiff neck, headache, emesis, diarrhea, or alcohol abuse.

The past medical history was of migraine headaches, gastritis secondary to aspirin, Sjögren's disease, multiple food allergies with malabsorption of wheat, corn, milk and citrus products, and hypothyroidism. Trials on pancreases in the past did not help the malabsorption. Her medications on admission were Inderal $10 \mathrm{mg}$ bid for migraine, Armor thyroid 2 gr. qAM, premarin $0.625 \mathrm{mg}$ qAM, Geritol multiple vitamin qAM, Stress tablet biw, 
Caltrate one qAM, Pepto-Bismol prn. Habits included heavy smoking until 1990 (age 71). The family history is positive for B12 deficiency. Her mother died of a cerebral vascular accident and her father committed suicide a few months later. There is no history of dementia in the family.

Physical examination with vital signs not given was unrevealing. It was noted the patient appeared to be a chronically ill, malnourished and volume depleted female. The mental status limited to "answering questions, but not always appropriately. She is not oriented to date or the day of the week." Neurological is reported as unremarkable, with no notation of myotatic irritability, hyperreflexia, or ataxia. Comment was made on "a lot of increased (muscle) tone".

Laboratory tests performed on admission showed that the electrolytes were normal except a serum potassium of $2.6 \mathrm{meq} / \mathrm{l}$ and Magnesium of $1.7 \mathrm{meq} / \mathrm{l}$, chemistry profile was unremarkable except a total protein of $6.5 \mathrm{Gm} / \mathrm{dl}$ and albumin of $3.7 \mathrm{gm} / \mathrm{dl}$. EKG and Chest $\mathrm{x}$-ray were unremarkable. A cranial MRI was read as "moderate atrophy, ventricular enlargement and ischemic white matter disease".

The initial diagnosis of Alzheimer's disease appeared based on the findings of the MRI alone. The husband was told that the patient was "not going to make it" and it was advised that he "allow them to let her go". The husband stated that he did not train with this ethic in medical school and searched for other possible diagnoses with a colleague. Five days later he insisted that a serum bismuth be done. The serum bismuth level from the 6 th hospital day was $242 \mu \mathrm{g} / \mathrm{L}$.

The diagnosis of bismuth toxicity was established. Subsequent history obtained revealed a seven-year use of Pepto-Bismol $30 \mathrm{cc}$ t.i.d. and h.s. to control her malabsorption (irritable bowel syndrome). At $1.04 \mathrm{mg}$ bismuth per ounce (30 cc), the patient had been ingesting $4.14 \mathrm{mg} / 24$ hours for about seven years.

The patient was started on penicillamine 250 mg q.i.d. pc from hospital day 9 to day 27 with no clinical improvement and a drop in serum bismuth to $128 \mu \mathrm{g} / \mathrm{L}$ on post hospital day 24 .

On post hospitalization day 22 the patient was transferred to a lower level of care and the husband was advised she would not recover and that she probably have to be placed in a nursing home. The author was asked to consult. At initial assessment she was in 4-point restraints, was cachectic, grossly confused, and spoke in a "word salad" with neologisms. She was oriented to her own first name only, with non-existent recent memory. She could not name her husband, recall the recent hospitalization, or name three common objects. Her mental testing revealed a MMS of $0 / 30 ; \quad$ OT $=1 / 12$; and NLT of $0 / 60$ (6). Neurologically, she had 4+/4+ DTR's with marked myotatic irritability, ankle myoclonus, and intermittent spasms of muscle groups. She was agitated to the point of trying to roll over the bed rails while in 4-point restraints.

Treatment consisted of pushing oral fluids to three liters per day, salt tablets and increasing titration of Cognex (Tacrine) to $20 \mathrm{mg}$-q.i.d. Within a few days her memory showed improvement and the myoclonic element subsided. On post hospitalization day 30 her serum bismuth had fallen to $90 \mu \mathrm{g} / \mathrm{L}$. She continued to improve over two weeks, and was discharged to home on the fifty-second post hospitalization day. Serum bismuth level on the 76th post hospitalization day was $14 \mu \mathrm{g} / \mathrm{L}$. Attempts to lower Cognex failed until the seventh month after onset of confusion. Eighteen months after hospitalization the patient has a normal mental status exam and lives at home with her husband leading a normal life.

\section{DISCUSSION}

Bismuth is a heavy metal that belongs in the class of lead, mercury, gold, silver and arsenic. All of these form strong covalent bonds with sulfhydryl groups of cellular proteins including vital enzymes. All are nephrotoxic and most have a strong affinity for the central nervous system tissue and will produce encephalopathy (e.g. "Mad Hatter syndrome" from mercury poisoning). There are four groups of medicinal bismuth compounds, of which bismuth subsalicylate (Pepto-Bismol) is in the most 
benign class; that is inorganic salts which are insoluble in water (3).

An "epidemic outbreak" of over 1,000 cases of bismuth encephalopathy were reported between 1973-1980 in France and Australia (2). Seventytwo of these intoxications were fatal. Most occurred with chronic high dose use of bismuth subnitrate or bismuth subgallate (3). Since 1980 case reports of bismuth encephalopathy have been infrequent. Nevertheless, there is higher risk of such cases because of the ready availability of bismuth salts and the increased use of Bismuth preparations in the treatment of Helicobacter pylori related gastric and duodenal ulcers (4).

In consideration of this specific patient, the diagnosis should have been suspected from the outset. Alzheimer's disease is an unrelenting progressive neurodegenerative disorder of insidious onset that leads to death on average 8 years after onset. Myoclonus is a very late and infrequent feature of Alzheimer's disease. Bismuth toxicity typically presents subacutely with mental changes of memory loss, psychosis and depression with a prominent background of ataxia, tremors, myoclonus and seizures (4).

In the present case the onset was about a week, myoclonus was a prominent feature, and although unstated in the initial HMO evaluation, it was obvious that the patient had ataxia. The present case is a testimony for the need of differential diagnosis evaluation of the demented patient. All too often the diagnosis of Alzheimer's is casually assigned. This practice must be discouraged. This case also points out the role of active treatment of the dementia aspects of patients. She was able to return to a normal life possibly months before anticipated because of the use of Tacrine. Finally, this case exemplifies why it is logical that bismuth becomes one of the metals tested in the "Heavy metal screen".

\section{References}

1. Bierer DW, Bismuth Subsalicylate: History, Chemistry, and Safety, Reviews of Infectious Disease 12 Supp (1990) S3-S8.

2. Gordon MF, Abrams RI, Rubin DB, Barr WB, Correa DD, Bismuth Subsalicylate toxicity as a case of prolonged encephalopathy with myoclonus, Movement Disorders 10(2) (1995) 220-222.

3. Serfontein WJ, Mekel R, Bismuth toxicity in man II. Research Communications in Chemical Pathology and Pharmacology, 26(2) (1979) 391411.

4. Shreeve DR, A double-blind study of tripotassium di-citrano bismuthate in duodenal ulcer, Postgraduate Medicine Journal 51 (Suppl 5) (1975) 33-36.

5. Slikkerveer A, De Wolff FA, Pharmacokinetics and toxicity of bismuth compounds, Medical Toxicology and Adverse Drug Experience 4(5) (1989) 303-323.

6. Summers WK, DeBoynton VL, Marsh GM, Majovski LJ, Comparison of seven psychometric instruments used for evaluation of treatment effect in Alzheimer's disease, Neuroepidemiology 9 (1990) 193-207.

7. Supino-Viterbo V, Sicard C, Risvegliato M, Rancurel G, Buge A, Toxic encephalopathy due to ingestion of bismuth salts: clinical and EEG studies of 45 patients, Journal of Neurology, Neurosurgery, and Psychiatry 40 (1977) 748752 . 